Faculdade de Ciências Econômicas da UFRGS
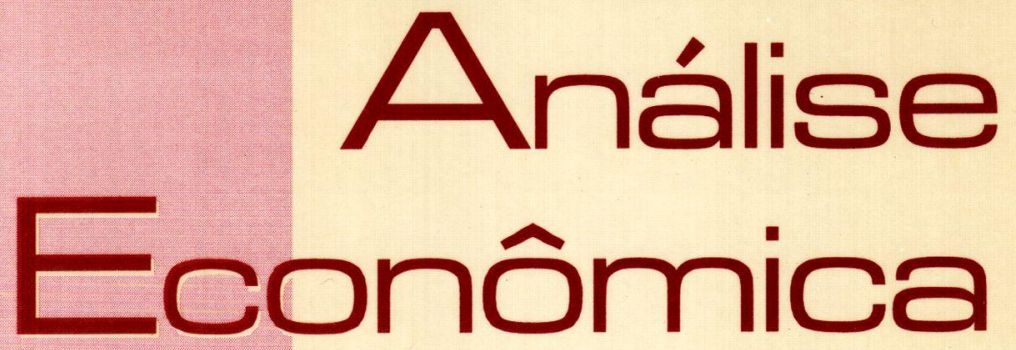

A Instabilidade Financeira dos Anos 90: Alqumas Implicações para as Economias Capitalistas Periféricas André Cunha e Daniela Prates

A Ampliação Recente da Participação Estrangeira no Sistema Bancário Brasileiro Maria Cristina Penido de Freitas

INFRA-ESTRUTURA dE INfORMAÇÕES E SISTEMA Nacional de InOvação

Eduardo da Motta e Albuquerque

Custo Social dos Recursos Hídricos em Bacias Hidrográlicas Internacionais: O Caso da Bacia do Paraná Jandir Ferrera de lima e José Carrera-Fernandez

Preferência pela liovidez e Escollha de Porrtólio

José Luis Oreiro

Eficiência, Objetivo e Coordenação da Política Macroeconômica no Período: 1974 - 1979

JoÃo Sicsú

Macroeconomia Moderna: Keynes e a ECONOMIA CONTEMPORÂNEA - Resenha

Simone Silva de Deos

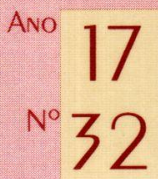


Universidade Federal do Rio Grande do Sul

Reitora: Proft. Wrana Maria Panizzi

Faculdade de Ciências Econômicas

Diretora: Prof ${ }^{a}$. Otilia Beatriz Kroeff Carrion

Centro de Estudos e Pesquisas Econômicas

Diretor: Prof. Fernando Ferrari Filho

Departamento de Ciencias Econômicas

Chefe: Prof Luiz Alberto Oliveira Ribeiro de Miranda

Curso de Pós-Graduação em Economia

Coordenador: Prof. Marcelo Savino Portugal

Curso de Pós-Graduaçāo em Economia Rural

Coordenador: Prof. Carlos Guilherme A. Mielitz Netto

Conselho edtrorial

Achyles B. Costa, Aray M. Feldens, Carlos A. Crusius, Carlos G. A. Mielitz Netto, Eduardo A. Maldonado Filho, Eduardo P. Ribeiro, Eugênio Lagemann, Fernando Ferrari Filho, Gentil Corazza, Marcelo S. Portugal, Nali J. Souza, Otilia B. K. Carrion, Paulo A. Spohr, Paulo D. Waquil, Pedro C. D. Fonseca, Roberto C. Moraes, Ronald Otto Hillbrecht, Stefano Florissi, Eleutério F. S. Prado (USP), Fernando H. Barbosa (FGV/RJ), Gustavo Franco (PUC/RJ), João R Sanson (UFSC), Joaquim P. Andrade (UnB), Juan H. Moldau (USP), Paul Davidson (Univ. of Tennessee), Werner Baer (Univ. of Illinois).

Comissão EDITORLAL

Eduardo Augusto Maldonado Filho, Fernando Ferrari Filho, Gentil Corazza, Marcelo Savino Portugal, Paulo Dabdab Waquil; Roberto Camps Moraes.

EDrTor: Gentil Corazza

Edrror Adjunto: Pedro Silveira Bandeira

SeCretaria: Laize Espindula.

REVISÃo DE TEXTos: Vanete Ricacheski.

Fundador: Prof. Antônio Carlos Santos Rosa

Os materiais publicados na revista Análise Econômica são da exclusiva responsabilidade dos autores. É permitida a reprodução total ou parcial dos trabalhos, desde que seja citada a fonte. Aceita-se permuta com revistas congêneres. Aceitam-se, também, livros para divulgação, elaboração de resenhas e recensões. Toda correspondência, material para publicação (vide normas na terceira capa), assinaturas e permutas devem ser dirigidos ao seguinte destinatário:

Prof, Gentul Corazza

Revista Análise Econômica - Av. João Pessoa, 52 CEP 90040-000 PORTO ALEGRE - RS, BRASIL

Telefones: 0xx (51) 316-3348 e 316-3440 - Fax: 0xx (51) 316-3990 rae@vortex.ufrgs.br

Análise Econômica

Ano 17, n. 32, setembro, 1999 - Porto Alegre

Faculdade de Ciências Econômicas, UFRGS, 1999

Periodicidade semestral, março e setembro.

ISSN 0102-9924

1. Teoria Econômica - Desenvolvimento Regional -

Economia Agrícola - Pesquisa Teórica e Aplicada -

Periódicos. I. Brasil.

Faculdade de Ciências Econômicas,

Universidade Federal do Rio Grande do Sul.

CDD 330.05

CDU 33 (81) (05) 


\section{Infra-estrutura de informações e sistema nacional de inovação: Notas sobre a emergência de uma economia baseada no conhecimento e suas implicações para o Brasil}

Eduardo da Motta e Albuquerque *

Sinopse: Este texto resenha as discussões atuais sobre a emergência de uma economia baseada no conhecimento (EBC), apresenta dados disponíveis e introduz uma discussão sobre as implicações para o caso brasileiro.

A emergência de uma economia baseada no conhecimento pressupõe a existência de um sistema de inovação maduro e a construção de uma infra-estrutura de informação, baseada na ampla utilização das tecnologias de informação e comunicação (TICs).

Para os países em desenvolvimento (países com sistemas de inovação imaturos) a emergência de uma EBC apresenta desafios e oportunidades: por um lado, amplia-se o hiato tecnológico em relação aos os países situados na fronteira tecnológica; por outro lado, a mudança de paradigmas tecnológicos coloca em cena "janelas de oportunidade" aos países periféricos.

Palavras-chave: economia baseada no conhecimento - sistema nacional de inovação - tecnologias de informação e comunicação.

Abstract: This paper discusses the emergence of a knowledge-based economy, presents available data, and introduces an evaluation of the Brazilian case.

The emergence of a knowledge-based economy is has as preconditions the existence of a mature innovation system and the formation of a information infrastructure. The information infrastructure is based upon the pervasive use of information and communication technologies.

For developing countries (countries with immature systems of innovation), the emergence of a knowledge-based economy presents challenges and opportunities. On the one hand, there is a widening of the technological gap vis-à-vis the developed countries. On the other hand, the paradigm shift opens "windows of opportunity" for developing countries.

Key words: knowledge-based economy - national systems of innovation technologies of information and communication.

\section{Introdução}

Este texto resenha as discussões atuais sobre a emergência de uma economia baseada no conhecimento (knowledge-based economy), apresenta dados disponíveis e introduz uma discussão sobre as implicações para o caso brasileiro.

* CEDEPLAR-UFMG e Pesquisador do Grupo "Economia da Inovação" do IE-UFRJ 
O significado e os impactos das mudanças em curso na economia mundial não podem ser subestimado. A Organização para a Cooperação e Desenvolvimento Econômico (OCDE), em documento recente, afirma que "as economias industriais estão no limiar de mudanças estruturais potencialmente radicais. Redes de comunicações e aplicações de multimídia interativa fornecem as fundações para a transformação das relações econômicas e sociais existentes em direção a uma 'sociedade de informação`. Tal sociedade é vista como resultante de uma mudança de paradigma nas estruturas sociais e nas relações sociais comparável à transformação imposta pela revolução industrial às sociedades então agrárias" (OECD, 1997a).

A dinâmica de permanente revolução nas bases técnicas da economia é uma das características do sistema capitalista (Schumpeter, 1942). Essas mudanças se distribuem no tempo, com a periódica emergência de novos paradigmas tecnológicos (Dosi, 1984), decorrentes de articulações complexas e multifacetadas entre os desenvolvimentos na ciência, a ação das firmas e a interação entre instituições de suporte ao desenvolvimento científico e tecnológico (Freeman \& Soete, 1997).

As tecnologias de informação e comunicação constituem o paradigma mais recente. Resultado de uma revolução tecnológica, em que o desenvolvimento e a generalização da microeletrônica é fundamental, as tecnologias de informação e comunicação viabilizam o surgimento de uma nova fase na economia: a emergência de uma economia baseada no conhecimento (EBC). ${ }^{1}$ Inúmeros estudos e documentos da $\mathrm{OCDE}$ e da Comissão Européia investigam diversos aspectos dessas mudanças (OECD, 1996b, 1997a, 1997b, 1997c; EC, 1996, 1997).

Para o Brasil (e outros países em estágio de desenvolvimento industrial e tecnológico similar), a emergência da EBC é plena de desafios e pode apresentar algumas novas oportunidades.

Por um lado, o principal desafio é claro: amplia-se o hiato tecnológico em relação à fronteira tecnológica internacional. Por outro lado, as oportunidades surgiriam em função das próprias características do novo paradigma e de características de períodos de transição tecnológicas (momento de surgimento de "janelas de oportunidade" para os países retardatários, segundo Perez \& Soete, 1988).

Porém o aproveitamento dessas "janelas de oportunidade" depende de esforços internos ao Brasil, especialmente através do desenvolvimento de capacidade de absorção de inovações desenvolvidas pelos países situados na fronteira tecnológica mundial. As condições para o aproveitamento das "janelas de

\footnotetext{
${ }^{1}$ Diferentes disciplinas atribuem nomes diversos para descrever esse fenômeno: sociedade da informação, por exemplo, é muito utilizada em estudos de sociólogos (Castells, 1996). Ao longo do presente texto, será utilizada a expressão economia baseada no conhecimento (OECD, 1996b).
} 
oportunidade" não são estáticas e a cada novo paradigma exigem investimentos internos mais sofisticados. A inexistência de um sistema nacional de inovação maduro no Brasil (Albuquerque, 1996) e as novas tarefas colocadas pela emergência da EBC delimitam a hipótese básica deste texto: a construção de uma infra-estrutura informacional torna-se um pressuposto para o amadurecimento do sistema de inovação do país.

Este texto é composto por seis seções. A próxima trata dos pressupostos de uma economia baseada no conhecimento. A terceira seção identifica a ampliação do hiato tecnológico entre os países avançados e o resto do mundo. A quarta seção avalia as oportunidades que a emergência da EBC pode oferecer aos países atrasados. A quinta seção discute as implicações para o caso brasileiro. A sexta seção apresenta as conclusões.

\section{Os pressupostos de uma economia baseada no conhecimento}

A tecnologia e o conhecimento sempre foram fontes do crescimento econômico. Recentemente, o papel do conhecimento tem sido mais destacado, uma expressão de seu peso crescente na dinâmica econômica. A OCDE considera que as suas economias "nunca foram tão dependentes da produção, distribuição e uso do conhecimento como agora" (OECD, 1996b, p. 9). O mesmo documento informa que "estima-se que, atualmente, nas principais economias da OCDE mais de 50\% de seus PNBs é baseado no conhecimento" (p. 9).

A identificação de uma EBC tem duas dimensões. Por um lado, reconhece que a capacitação humana, o aprendizado e o conhecimento estão na base do desenvolvimento de todas as sociedades humanas. Por outro lado, enfatiza novas características da economia a ponto de caracterizar uma nova era histórica (Foray \& Lundvall, 1996, p. 12).

À luz das discussões sobre a economia da tecnologia e da inovação, a emergência de uma $\mathrm{EBC}$ tem dois pressupostos básicos: 1) a existência de sistemas nacionais de inovação maduros; 2) a disseminação das tecnologias de informação e comunicação. Uma infra-estrutura de informação passa a ser um componente crucial de um sistema nacional de inovação.

\subsection{Sistemas de inovação maduros}

O mercado tem profundas limitações para gerar um componente essencial da dinâmica capitalista: a inovação tecnológica. A livre operação das forças de mercado levaria a uma situação de subinvestimento em atividades inovativas (Arrow, 1962).

Para superar essas limitações, instituições fora da lógica do lucro foram 
construídas ao longo do tempo, compondo a base da complexa máquina capitalista moderna (Nelson, 1990). Sintetizando tanto as limitações do mercado como as contribuições das instituições públicas, o conceito de sistema nacional de inovação descreve quais as articulações necessárias para a endogenização do progresso tecnológico.

Sistema nacional de inovação é uma construção institucional, produto seja de uma ação planejada e consciente ou de um somatório de decisões não planejadas e desarticuladas, que impulsiona o progresso tecnológico em economias capitalistas complexas (Freeman, 1988; Nelson, 1993). Através da construção desse sistema de inovação se viabiliza a realização de fluxos de informação necessários ao processo de inovação tecnológica. Esses arranjos institucionais envolvem as firmas, redes de interação entre empresas, agências governamentais, universidades, institutos de pesquisa, laboratórios de empresas, atividade de cientistas e engenheiros. Arranjos institucionais que se articulam com o sistema educacional, com o setor industrial e empresarial, e também com as instituições financeiras completando o circuito dos agentes que são responsáveis pela geração, implementação e difusão das inovações.

O papel das universidades, das instituições de pesquisa e do conjunto da estrutura educacional para a manutenção dos fluxos de informação constitutivos de um sistema de inovação maduro indica uma metamorfose que é um prérequisito da nova fase. O National Science Foundation (NSF, 1996) estima que o total de cientistas e engenheiros empregados em atividades de $\mathrm{P} \& \mathrm{D}$ nos Estados Unidos alcançou a cifra de 962.700 pessoas em 1993 (cerca de 1\% da força de trabalho do país). O total de cientistas e engenheiros empregados em atividades não-acadêmicas atingiu a 3.502 .000 (1.749.000 cientistas e 1.753.000 engenheiros), cerca de $3 \%$ da força de trabalho em 1992.

Um sistema de inovação sofistica a divisão tecnológica de trabalho, fornecendo às firmas oportunidades tecnológicas de forma persistente.

Esse arranjo complexo está por trás de avanços cruciais da revolução tecnológica da informação e da comunicação. O pioneiro computador ENIAC foi financiado pela Força Aérea norte-americana. A Internet é produto de uma divisão do Departamento de Defesa dos Estados Unidos (ARPA), que criou durante os anos sessenta a ARPANET e do NSF, que criou em meados da década de oitenta a NSFNET. A NSFNET constituiuse na espinha dorsal para o desenvolvimento da Internet como é conhecida hoje (Varian, 1996).

Num certo sentido, a revolução da informação e da comunicação é um produto do amadurecimento dos sistemas nacionais de inovação. O desenvolvimento das tecnologias de informação e comunicação, por sua vez, muda a estrutura das economias desenvolvidas, com os investimentos sendo progressivamente dirigidos para bens e serviços de alta tecnologia: "computadores e 
equipamentos relacionados são os componentes de investimentos tangíveis que têm crescido mais rapidamente." (OECD, 1996b, p. 10).

\subsection{As tecnologias de informação e comunicação}

Para Foray \& Lundvall (1996, p. 13-14) a revolução da informação e da comunicação não é equivalente à EBC. Para eles, as tecnologias de informação e comunicação “fornecem à EBC uma base tecnológica nova e diferente, que muda radicalmente as condições para a produção e distribuição de conhecimento, assim como a sua vinculação com o sistema de produção".

Foray \& Lundvall sistematizam os efeitos de longo e médio prazo das tecnologias de informação e comunicação (TICs).

A longo prazo, os efeitos das TICs implicam "um novo potencial de ganhos de produtividade no processo de geração, distribuição e exploração do conhecimento". É possível identificar três caminhos para tanto: a) o aumento do ritmo da inovação em termos de desenvolvimento de novos produtos e processos (que envolve a aceleração da taxa de obsolescência técnica); b) a crescente habilidade de gerar opções tecnológicas; c) o maior poder das redes eletrônicas como instrumentos de pesquisa (p. 14).

A médio prazo, há um processo mais complexo: problemas derivados do desencontro (mismatch) entre tecnologias e instituições. Segundo Foray \& Lundvall, "indivíduos confrontando-se pela primeira vez com computadores e firmas utilizando tecnologias de automação, assim como com tecnologias de redes e com informação sistêmica, devem esquecer como operavam nas velhas formas, aprender novos hábitos e colocar em prática formas completamente novas de organização. Isso requer uma aceleração e uma ampliação dos processos de aprendizado em muitos níveis da sociedade, envolvendo firmas, sistemas de treinamento e educação e instituições formais como direitos de propriedade intelectual e comitês de estabelecimento de padrões. Espera-se, com base em experiências históricas análogas, que apenas após um longo período de aprendizado institucional e mudanças organizacionais se realizará plenamente o potencial de fortalecimento da produtividade da revolução das TICs" (p. 14).

\subsection{Infra-estrutura informacional}

O desenvolvimento das telecomunicações e a convergência entre as indústrias de comunicação, informática e provedores de informação (banco de dados etc.) permitem que uma nova infra-estrutura de informações seja identificada (e quantificada, como se verá na próxima seção). 
Uma infra-estrutura informacional não é novidade: na emergência da revolução industrial, as bibliotecas, a imprensa, os correios, etc. forneciam essa infra-estrutura (Thompson, 1995). Ao longo do desenvolvimento da história do capitalismo, invenções como o telégrafo, o telefone, o rádio e a televisão, os satélites de comunicação etc. aprimoraram essas redes. Ao mesmo tempo em novos setores industriais surgiam, esses progressos nos meios de comunicação possibilitaram mudanças nas estruturas das firmas e nas formas de relacionamento e interação entre elas. Os fluxos de informação constitutivos dos sistemas de inovação ampliavam-se e circulavam com mais rapidez. ${ }^{2}$

A novidade neste momento de emergência da EBC é dupla. Por um lado, a infra-estrutura de informação nunca foi tão abrangente, constituindo-se em uma das bases da nova fase da internacionalização da produção e das relações financeiras e comerciais conhecida como globalização. Por outro lado, a infraestrutura informacional passa a ser um elemento crucial para a dinâmica econômica, constituindo-se em um tópico importante para avaliação dos sistemas de inovação contemporâneos.

A atenção devotada pela OCDE à infra-estrutura de informação global é uma constatação desse papel (OECD, 1996a, 1997a).

A infra-estrutura informacional pode ser decomposta em cinco elementos: a) indústrias de tecnologias de informação (semicondutores, computadores, software e serviços relacionados); b) indústrias de tecnologias de comunicação; c) infra-estrutura de telecomunicações (telefonia, comunicação de dados, rádio e televisão, redes); d) computadores em rede (Internet, Intranets); e) indústrias editorial e gráfica.

Como será visto na próxima seção, esses recursos informacionais não estão distribuídos de forma simétrica à escala mundial, sendo que a sua concentração nos países mais desenvolvidos sinalizam para a ampliação do hiato tecnológico entre os países desenvolvidos e o resto do mundo.

O papel da infra-estrutura de informação coloca uma nova questão quanto a serviços universais e acesso público, requalificando-os para a nova fase da economia. Um país (ou região) inadequadamente provido desses serviços e conexões perderá capacidade inovativa. Por isso, governos e instituições internacionais têm se debruçado com tanto interesse sobre essa questão (OECD, 1996a).

Por sua vez, esse novo papel coloca novas questões para a ação do setor público, seja em nível de regulação seja em nível de provimento de serviços e recursos. As exigências de coordenação e padronização internacionais são enor-

\footnotetext{
${ }^{2}$ Chandler (1990, p. 26) relaciona a revolução nos transportes e nas comunicações (com o aparecimento do telégrafo) à revolução nas formas de produção e distribuição entre as grandes empresas norte-americanas e européias no início do século. Arrow (1996) discute o impacto das novas tecnologias de informação sobre a estrutura industrial nesse fim de século.
} 
mes, envolvendo questões técnicas (conectividade, protocolos, normas, etc.), questões econômicas (aquisições e fusões de empresas estratégicas, novo formato de redes de firmas, etc.) e institucionais (segurança para dados e negócios nas redes, novas formas de proteção à propriedade intelectual, etc.).

\subsection{Definição de uma economia baseada no conhecimento}

Uma economia baseada no conhecimento pode ser definida por: a) preexistência de um sistema nacional de inovação maduro; b) destaque para o papel da infra-estrutura de conhecimento como componente desse sistema de inovação (Smith, 1997); c) construção de uma infra-estrutura informacional, como uma conseqüência da revolução das TICs; d) sofisticação da divisão de trabalho, com a participação de parcelas expressivas da força de trabalho em atividades de produção, difusão e transmissão de conhecimentos.

Características importantes da EBC são: a) nova dinâmica na formação de conhecimento tácito e codificado; b) importância crescente das redes de conhecimento; c) aceleração dos processos de aprendizado interativo (Foray \& Lundvall, 1996, p. 13).

Os estímulos para a codificação do conhecimento (Cowan \& Foray, 1997), combinados com as facilidades de armazenamento e acesso a dados, apresentam uma das mais importantes fontes de oportunidades para os países mais atrasados. A existência de informações científicas e tecnológicas disponíveis enfatiza a necessidade dos países desenvolverem capacidades de absorção desses conhecimentos, o que pode se tornar em uma poderosa ferramenta para o crescimento econômico.

A importância crescente das redes de conhecimento, enfatizando o papel da infra-estrutura informacional, pode contribuir para redefinir o papel do setor público, na medida em que a Comissão Européia chega a especular sobre um eventual papel estratégico dos serviços públicos de informação: poderiam eles se transformar em um dos novos motores do crescimento econômico? (EC, 1997, p. 26$30)$.

Os múltiplos impactos da emergência da economia baseada no conhecimento se combinam com outros fenômenos contemporâneos e tornam a análise do seu significado complexa: a globalização das atividades financeiras, maior internacionalização das atividades de $\mathrm{P} \& \mathrm{D}$, a ampliação do espectro de ação das grandes corporações transnacionais, a existência de pressões sobre a ação sindical e as reformas nas estruturas de bem-estar social dos países mais desenvolvidos. Alguns desses fenômenos podem ser diretamente articulados com a revolução das tecnologias de informação e comunicação (globalização das atividades financeiras, internacionalização de atividades de $P \& D$ ), en- 
quanto outros teriam fatores determinantes distintos, mais políticos e decorrentes de correlação de forças nos cenários nacionais e internacionais (pressões sobre movimentos sindicais, desregulamentação etc.).

A emergência da EBC, porém, se relaciona com um processo de afastamento da fronteira tecnológica mundial dos países mais atrasados, uma característica em comum com o início de outras novas fases da economia mundial (Freeman \& Perez, 1988).

\section{Ampliação do hiato tecnológico}

A concentração da riqueza econômica, dos recursos industriais, tecnológicos e científicos na "Tríade" (Estados Unidos, Europa e Japão) é bem-documentada (World Bank, 1997; European Commission, 1996). Neste período de transição tecnológica essa diferenciação se amplia, pois a concentração dos mercados de tecnologias de informação e dos recursos informacionais é ainda maior do que a dos recursos econômicos "tradicionais".

A Tabela 1 apresenta dados gerais desse cenário. A participação dos países desenvolvidos (incluídos na OCDE) nos mercados de tecnologias de informação $(91,6 \%$ em 1994) é superior à sua participação em termos de PNB $(80,8 \%$ em 1994).

Para localizar o caso brasileiro, os dados para 1995 foram os seguintes: a) população: $2,8 \%$; b) PNB: $2,4 \%$ (World Bank, 1997); c) mercado de tecnologias de informação: $1,1 \%$ do total mundial (OECD, 1997c). Ou seja, o Brasil se enquadra no conjunto de países cuja participação no mercado mundial de TICs é inferior à sua participação de seu PNB. ${ }^{3}$

Tabela 1: População, Produto Nacional Bruto (PNB) e Mercado de Tecnologias de Informação (TIs) (\% do total mundial)

OCDE RESTO DO MUNDO

\begin{tabular}{ccc}
\hline & & \\
1987 & & \\
Pop. & 17,9 & 82,1 \\
PNB & 78,7 & 21,3 \\
TIs & 93,7 & 6,3 \\
& & \\
1994 & & \\
Pop. & 17,4 & 82,6 \\
PNB & 80,8 & 19,2 \\
TIs & 91,6 & 8,4 \\
\hline
\end{tabular}

FONTE: OECD, 1997c.

${ }^{3} \mathrm{O}$ Brasil produz $0,8 \%$ dos artigos científicos mundiais e $0,06 \%$ das patentes registradas no UFPTO (uma proxy das patentes mundiais) 
A Tabela 2 apresenta esses dados de outra forma, detalhando a distribuiçãc do mercado mundial de TICs por região. A posição da América Latina é apresentada: 2\% do mercado mundial em 1995.

Tabela 2: Mercado mundial de tecnologias de informação, desagregação por região (\%)

$1985 \quad 1990 \quad 1995$

$\begin{array}{lrcr}\text { América do Norte } & 59,2 & 41,9 & 43,5 \\ \text { América Latina } & 1,5 & 1,6 & 2,0 \\ \text { Europa Ocidental } & 22,1 & 33,6 & 28,3 \\ \text { Eur. Or., Oriente } & & & \\ \text { Médio e África } & 3,1 & 2,5 & 2,6 \\ \text { Ásia (Reg. Pacífico) } & 14,0 & 20,4 & 23,7\end{array}$

FONTE: OECD, $1997 \mathrm{c}$.

A Tabela 3 aborda a produção de bens de tecnologias de informação e comunicação. Segundo a $\mathrm{OECD}$, esses bens são equipamentos de processamento de dados, equipamentos de escritório, radar e telecomunicações, áudio, vídeo e componentes (OECD, 1997c, p. 197). Grosso modo, esses bens envolvem as partes industriais da infra-estrutura informacional apresentada no tópico anterior (pontos "a" e "b")

Tabela 3: Produção mundial de bens de tecnologias de informação e comunicação (valor e \%)

$\begin{array}{ccc}1985 & 1990 & 1995 \\ \text { US\$ bil. }(\%) & \text { US\$ bil. (\%) } & \text { US\$ bil. (\%) }\end{array}$

\begin{tabular}{lrrr}
\hline & & & \\
EUA & $150,042(44,6)$ & $169,638(29,0)$ & $201,093(28,3)$ \\
Japão & $81,509(24,2)$ & $169,855(29,1)$ & $216,350(30,4)$ \\
Alemanha & $17,133(5,1)$ & $35,352(6,1)$ & $30,329(4,3)$ \\
OCDE 18 & $306,668(91,1)$ & $494,331(84,7)$ & $556,843(78,3)$ \\
EDA $\left(^{*}\right)$ & $21,358(6,3)$ & $68,487(11,7)$ & $126,068(17,7)$ \\
Brasil & $4,140(1,2)$ & $11,057(1,9)$ & $12,656(1,8)$
\end{tabular}

FONTE: OECD, $1997 \mathrm{c}$, p. 50

(*) Taiwan, Hong Kong, Malásia, Coréia do Sul, Cingapura e Tailândia

Note-se que a participação dos países fora da OCDE é maior nesse caso: em 1994 as economias dinâmicas da Ásia e o Brasil somaram 19,5\% da produção mundial. $O$ recuo da participação relativa dos Estados Unidos parece ter ocorrido às custas do crescimento da produção dos bens de TICs nas economias dinâmicas da Ásia. A participação do Brasil (1,8\% em 1994) se aproxima mais aqui do que no caso anterior da sua participação em termos de PNB.

Em termos de infra-estrutura de telecomunicações, a diferença entre os países avançados e os atrasados é expressiva. Segundo a Comissão Européia (1996), utilizando a teledensidade como indicador, o contraste é claro: o número para os 
países industrializados é 48, para os países de renda média aproximadamente $10 \mathrm{e}$ para os países menos desenvolvidos 1,5. A média mundial é 11,5. Dantas (1996, p. 92), a partir de estatísticas da OCDE de 1992, apresenta dados para telefones e televisões: os países desenvolvidos detinham uma média de 34 aparelhos de televisão e 35 linhas de telefones por grupo de 100 habitantes, enquanto os países em desenvolvimento tinham respectivamente 2 e 1,5 .

Outra comparação relevante, segundo a Comissão Européia (1996) é a relação de PCs por 100 habitantes: 18 para os países de alta renda, 2,3 para os de renda média e 0,01 para os países de baixa renda. Nessa distribuição, há uma diferenciação no interior dos países de alta renda: os Estados Unidos têm 32,5 PCs por 100 habitantes, enquanto os 14 principais países da Europa alcançam uma média de 14 PCs por 100 habitantes e o Japão 15 por 100 (OECD, 1997c, p. 21).

A Tabela 4 completa o panorama dos dados relacionados aos cinco componentes da infra-estrutura informacional, apresentando a distribuição dos servidores da Internet: a concentração atualmente existente nos Estados Unidos é brutal, atingindo em 1997 a $62,6 \%$ do total. ${ }^{4}$

Hawkins (1995, p. 43) considera que as capacidades de P\&D e produção de TICs

Tabela 4: Servidores da Internet por região (\%)

\begin{tabular}{lc}
\hline Região & Participação (\%) \\
\hline \multicolumn{1}{l}{$\quad$ JAN. 1995 } & \\
Estados Unidos/Canadá & 70 \\
Europa Ocidental & 21 \\
Região do Pacífico & 4 \\
Ásia & 3 \\
Outros & 2 \\
$\quad$ & \\
$\quad \ldots$ 1996 & 66 \\
América do Norte & 16 \\
Europa & 14 \\
Ásia/Reg. Pacífico & 4 \\
Outros & \\
$\quad$ JAN. 1997 & 62,6 \\
Estados Unidos & 3,1 \\
Canadá & 3,6 \\
Japão & 3,4 \\
Alemanha & 2,6 \\
Reino Unido &
\end{tabular}

Fontes: OECD (1997c), The Economist, Mansell et all (1996)

${ }^{4}$ Mansell et al. (1996, p. 5-6) apresenta o desafio da construção da Internet-II nos Estados Unidos (envolvendo universidade e a NSF, para fugir do congestionamento e privatização da Internet), em um contexto em que o conjunto dos países em desenvolvimento ainda luta para ter acesso à Internet de "primeira geração". A Internet-II operará com $622 \mathrm{Mbits} / \mathrm{s}$, enquanto a Embratel anuncia a instalação de uma rede de alta velocidade no Brasil: $34 \mathrm{Mbits/s} \mathrm{(Gazeta} \mathrm{Mercantil,} \mathrm{10/03/1998,} \mathrm{p.} \mathrm{C-2).}$ 
estão altamente concentradas nos países que constituem o G-7. Dados recentes da ITU, por exemplo, mostram que, dos aproximadamente US $\$ 96$ bilhões de faturamento provenientes das 16 maiores empresas que desenvolvem equipamentos de telecomunicações, mais de $70 \%$ são gerados por somente sete empresas localizadas em seis países (Estados Unidos, Alemanha, Canadá, França, Suécia Japão). A conclusão de Hawkins (p. 45) é a de que "a dimensão global da infra-estrutura de TIC está se desenvolvendo em torno de centros regionais de poder".

Qual o significado do panorama delineado por esses dados? Em primeiro lugar, atesta-se uma concentração de recursos informacionais nos países já desenvolvidos. O hiato tecnológico entre desenvolvidos e o resto amplia-se. Em segundo lugar, dado o caráter estratégico das TICs para a dinâmica econômica atual, a médio prazo essa concentração de recursos pode determinar um novo tipo de "círculo vicioso", podendo significar novas perdas de espaço.

A Tabela 5 justapõe os dados para $P \& D$ e para software e serviços relacionados à computação. ${ }^{5}$ É conhecida a restrição dos investimentos em $\mathrm{P} \& \mathrm{D}$ nos países em desenvolvimento (no caso do Brasil, esses gastos estão em torno de $0,8 \%$ ). Os dados para os Estados Unidos apontam para o vigor dos gastos nesses setores: 2,4\% do PNB para o P\&D, 2,7\% para software e serviços.

Tabela 5: Gastos com P\&D e com Software e serviços relacionados a computadores ( $\%$ do PNB), países selecionados

\begin{tabular}{lcc}
\hline & P\&D & Software \\
\hline & & \\
Estados Unidos & $2,4(1995)$ & $2,7(1995)$ \\
Japão & $2,7(1993)$ & $1,3(1995)$ \\
Canadá & $1,5(1994)$ & $1,1(1995)$ \\
França & $2,4(1993)$ & $0,9(1995)$ \\
Holanda & $1,8(1992)$ & $0,7(1992)$ \\
Finlândia & $2,2(1992)$ & $0,8(1992)$ \\
& & \\
\hline
\end{tabular}

Fonte: OECD (1997d), NSF (1996), EC (1994)

Esses dados (ao lado de outros como a distribuição de computadores por habitante e servidor da Internet) apontam para o crescimento do hiato mesmo entre os Estados Unidos e o restante dos países desenvolvidos. Documento da Comissão Européia reconhece essa realidade, identificando que as vantagens comparativas da Europa estão nos produtos e técnicas do passado, com uma especialização limitada nos setores de alta demanda e alta tecnologia (EC, 1997, p. 37).

\footnotetext{
${ }^{5}$ Os dados de gastos com software são importantes, pois buscam preencher uma lacuna nos indicadores mais clássicos de inovação tecnológica. Patel \& Pavitt (1995) têm insistido em que uma das principais fraquezas das estatísticas de patentes é o fato delas não captarem a produção de software.
} 


\section{Oportunidades abertas pela emergência da economia baseada no conhecimento}

Períodos de transição tecnológica, com a emergência de novos paradigmas, são também períodos em que se abrem "janelas de oportunidade", tanto nos países avançados (para novas firmas dos setores industriais emergentes) como nos países atrasados (para firmas e para os próprios países).

A movimentação da fronteira tecnológica internacional é determinada pela sucessão de paradigmas tecnológicos (Dosi, 1984; Freeman \& Perez, 1988). Inovações radicais estabelecem as bases de constituição de um novo paradigma tecnológico (Freeman, 1994). A carga de incerteza presente no processo de definição de um novo paradigma é enorme. Estabelecido um novo paradigma, trajetórias tecnológicas serão estabelecidas, fundamentalmente a partir de inovações incrementais. $O$ estabelecimento de um novo paradigma, portanto, cria um enorme conjunto de oportunidades tecnológicas.

É justamente quando do surgimento de novos paradigmas que se abrem "janelas de oportunidade" (Perez \& Soete, 1988) aos países atrasados. Por que surgem essas "janelas de oportunidade" ? Segundo Dosi (1984, p. 93-94), as condições de apropriabilidade das inovações variam ao longo das "fases" do paradigma. $\mathrm{Na}$ emergência de um novo paradigma, caracterizada por uma alta taxa de natalidade e mortalidade de "novas firmas schumpeterianas", oligopólios temporários seriam estabelecidos; enquanto nas trajetórias estabelecidas, a apropriabilidade privada é mais forte e as estruturas oligopolísticas mais estáveis. Essa variação nas condições de apropriação das inovações ao longo das fases dos paradigmas ilustra a variação nas condições de difusão das inovações (mais fácil quando a apropriação é mais fraca). Essas condições, válidas para os processos internos de um país, se repetem na arena internacional, fundamentando o aparecimento das "oportunidades" para as firmas dos países atrasados.

Em suma, a movimentação da fronteira tecnológica internacional apresenta dois aspectos contraditórios: em primeiro lugar, ao ampliar o hiato tecnológico entre as nações (avaliado na seção anterior), introduz a possibilidade de catching up; em segundo lugar, dadas as mudanças nas condições de apropriabilidade que determina, abre "janelas de oportunidade" para os países retardatários.

Finalmente, a movimentação da fronteira internacional, decisiva para o surgimento de oportunidades aos países retardatários, apresenta um problema adicional (e decisivo) para eles: a amplitude e a qualidade do esforço interno a ser realizado também varia de forma dinâmica, crescendo à medida que os paradigmas se sucedem. Uma singularidade do presente paradigma é demonstrada pela necessidade da construção de uma infra-estrutura informacional. 
O novo paradigma (EBC) tem outra especificidade que é a maior disponibilidade de informações científicas e tecnológicas, dada a maior codificação do conhecimento e a maior facilidade técnica de acesso a informações relevantes. $O$ peso maior da ciência na definição do presente paradigma, assim como a ampliação da internacionalização da comunidade científica, também contribui para uma maior facilidade de difusão de elementos cruciais para a dinâmica inovativa. ${ }^{6}$

Outro elemento que fornece possibilidades para países atrasados são os desdobramentos da evolução tecnológica nos países mais avançados. A Tabela 6 mostra as mudanças ocorridas no mercado mundial de TIs. Destacam-se três mudanças: a) a queda da participação dos computadores de grande porte; b) a ampliação da parcela detida por PC's e workstations; c) a regressão do peso do hardware em geral, pois software e serviços relacionados à computação passaram a deter mais da metade do mercado de TIs (OECD, 1997c).

Duas consequiências importantes dessas mudanças são relevantes para a presente discussão. Em primeiro lugar, há uma queda de custos (do mainframe para os PCs) acompanhada por um extraordinário aumento de capacidade de processamento de dados. Como o Information Technology Outlook 1997 (OECD, 1997 c, p. 25) sugere, os países mais atrasados podem ter "saltado" a fase dos computadores de grande porte. Em segundo lugar, o peso crescente de software e serviços relacionados à computação, que possuem um caráter muito mais específico a mercados locais (em função de língua, características legais, instituições diferenciadas etc.), reforça a abertura da "janela de oportunidade" nesse setor.

Cassiolato (1997, p. 33-34), em uma avaliação voltada para as tecnologias da telemática, afirma que, em países em desenvolvimento, há firmas e instituições que estão adotando novas tecnologias de telemática de forma tão eficiente ou mais (automação bancária no Brasil, segundo Cassiolato, 1992) do que em países avançados. Nesse caso, com investimentos apropriados é possível que exista uma "vantagem do atraso". Cassiolato cita uma pesquisa que mostrou que "países retardatários com um início tardio de adoção, um rápido crescimento da produção e investimento e uma pequena base instalada da tecnologia inferior obtiveram um desempenho superior ao de muitos países avançados" (p. 33). Os países avançados se encontrariam trancados pelos altos custos de mudança da tecnologia antiga para a mais nova.

As "janelas de oportunidade", porém, pressupõem um esforço interno aos países atrasados: o desenvolvimento de capacidade de absorção é o elemento determinante. ${ }^{7}$ Não há um desenlace previamente determinado nesse caso.

\footnotetext{
${ }^{6}$ Essas características intrínsecas à EBC são contrapostas por esforços dos Estados Unidos de fortalecimento da proteção à propriedade intelectual na arena internacional (David, 1992), uma forma de criação de barreiras artificiais à difusão agora tecnicamente mais fácil

${ }^{7}$ Em outro trabalho (Albuquerque, 1997) é exposto o papel da capacidade de absorção e sua relação com o amadurecimento de um sistema nacional de inovação
} 


\section{Implicações para o Brasil}

O resultado final da disputa entre desafios e possibilidades depende do desenvolvimento de investimentos internos ao país, responsáveis pelo aprimoramento da capacidade de absorção do país. $O$ ponto de partida da análise é problemático: conforme apresentado na seção três, o hiato tecnológico cresce em relação aos países de fronteira (e em relação aos Estados Unidos em especial). Deriva-se daí um problema novo, específico ao paradigma atual que é o atraso na construção da infra-estrutura informacional. ${ }^{8}$

A especificidade do caso brasileiro é a combinação desse "novo" atraso (infra-estrutura informacional) com os "velhos" atrasos (educação, disseminação da formação universitária, etc.). ${ }^{9} \mathrm{O}$ desafio atual é a necessária combinação da superação desse duplo atraso: por exemplo, o analfabetismo informático não pode ser superado sem a elevação do nível médio de educação do país, sem a generalização do ensino de matemática etc.

Em síntese, o Brasil tem o desafio de completar o processo de construção de um sistema nacional de inovação em um momento em que a construção de uma infra-estrutura informacional passa a ser um componente essencial desse sistema.

A inter-relação dessas duas dimensões é crucial. Para o desenvolvimento da capacidade de absorção, são necessários investimentos pesados na educação: para se ter acesso ao conhecimento disponibilizado nas redes informacionais é necessário que se saiba o que se procura, qual a informação relevante etc. Ao mesmo tempo, sem uma infra-estrutura adequada, tal acesso, tecnicamente possível, fica inviabilizado. Um papel que a infra-estrutura científica construída no país pode desempenhar é o de atuar como uma "antena" para absorver conhecimentos gerados nos países mais avançados, um primeiro passo para adaptá-los às condições nacionais e para iniciar um processo de aperfeiçoamentos incrementais (Albuquerque, 1998). Novamente, esse papel depende crucialmente de uma infra-estrutura adequada.

Por tudo isso, é fundamental insistir na necessária combinação da construção da infra-estrutura informacional e o amadurecimento do sistema de inovação.

A discussão da seção 2.3 indicou cinco componentes da infra-estrutura informacional. Não parece ser necessária a construção de todos os componen-

\footnotetext{
${ }^{8}$ Esse atraso é importante, como discutido. Porém é necessário demarcar a posição intermediária do Brasil (entre os países menos desenvolvidos e os mais desenvolvidos). Assim como a situação do sistema nacional de inovação foi caracterizada como "imatura" (em oposição a inexistente e maduro), os dados do mercado de TICs, desenvolvimento da rede de telecomunicações etc também situam o país numa categoria intermediária. Coerente com o estágio de construção do sistema de inovação.

${ }^{9}$ Para uma discussão abrangente da condição periférica do Brasil, ver Lemos (1988)
} 
tes dessa infra-estrutura para que um país se atualize e aproveite as oportunidades da EBC. No caso brasileiro, há setores em que a entrada é difícil (indústria microeletrônica, por exemplo) e custosa. Partes da infra-estrutura informacional podem ser obtidas através de um uso inteligente da divisão internacional de trabalho. As conclusões do ECIB, por exemplo, apresentam sugestões nessa linha (Coutinho \& Ferraz, 1994). A discussão detalhada desse aspecto ultrapassa os objetivos deste texto. $\mathrm{O}$ importante para a presente discussão é apontar os elementos que parecem ser indispensáveis para o país construir a sua infra-estrutura informacional: a produção de software, a prestação de serviços relacionados a computação e a construção de infra-estrutura de telecomunicações. ${ }^{10}$

\subsection{Software}

As possibilidades para a área de software foram analisadas em outros textos (Pondé, 1993; Evans et al., 1992; Lucena, 1994; Cassiolato, 1997). ${ }^{11}$

Segundo Perez \& Soete (1988), os custos de entrada podem ser desagregados em quatro componentes: a) o conhecimento científico e tecnológico relevante $(\mathrm{S})$; b) investimentos de capital $(\mathrm{I})$; c) experiência gerencial e organizacional (E); d) custos locacionais $(X)$.

O conhecimento científico e tecnológico acumulado tem um desenvolvimento razoável neste setor: o conhecimento público é relevante (Lucena, 1993; Pondé, 1993). A empresa inovadora deve arcar com um custo pequeno para fechar o hiato entre o conhecimento público disponível e o total de conhecimento relevante necessário para a firma entrar nesta tecnologia.

Quanto aos outros custos de entrada, o custo de capital fixo (I) não é proibitivo, dada a tendência de baixa de custo dos equipamentos de informática (da ordem de milhares de dólares). Aliás, neste setor a pequena empresa tecnologicamente inovadora pode ter um papel extremamente importante. Dado o legado da experiência anterior na indústria de informática, existe experiência acumulada e os custos a ela relacionados (E) são baixos (Villaschi, 1992). $O$ problema maior estaria nos custos locacionais $(X)$, em especial devido às limitações do mercado interno (Schware, 1992). Estas limitações podem ser superadas, inicialmente, pela conquista de parcelas do mercado internacional

\footnotetext{
${ }^{10} \mathrm{O}$ aspecto industrial é importante, mas para o periodo atual a preservação de capacidade produtiva em bens eletrônicos de consumo pode ser suficiente para garantir um processo de capacitação tecnológica de firmas no país que as qualifiquem para adiante avançar para produtos mais complexos (como foi o processo coreano, segundo Amsden, 1989), tanto de computação como de comunicação.

${ }^{11}$ Esta parte do texto baseia-se em Albuquerque (1998), em que as possibilidades de entrada na indústria de software foram contrastadas com as possibilidades nas áreas de microeletrônica, quimica fina e biotecnologia. Apenas em biotecnologia foi avaliada existir uma possibilidade de aproveitamento de oportunidade comparável à da área de software.
} 
e pela abertura de espaço no país, através do desenvolvimento de software específicos para as atividades econômicas internas, ao longo de um processo de modernização consistente do parque brasileiro. ${ }^{12}$

Portanto, na área de Software a contribuição do setor científico é relevante e, no geral, os outros custos de entrada não são proibitivos.

Essas características de custo do setor de software, o acúmulo de conhecimento especializado nas universidades e os exemplos internacionais indicam que não é descartada a possibilidade de crescimento do número de pequenas firmas tecnologicamente inovadoras através do processo de spin-off.

\subsection{Infra-estrutura de telecomunicações}

A política para o setor de telecomunicações desempenha um papel crucial para a construção da infra-estrutura informacional. O Estudo da Competitividade da Indústria Brasileira (ECIB) encontrou nesse setor um quadro de "virtual estagnação da capacidade instalada e degradação da qualidade dos serviços." (Coutinho \& Ferraz, 1994, p. 147). O ECIB avaliou que tal situação ainda tinha se constituído em um entrave à competitividade, mas poderia vir a "comprometer as condições sistêmicas de longo prazo da competitividade, que são justamente intensivas nesse tipo de serviços modernos" (p. 147). ${ }^{13}$

A proposta de privatização do setor em curso no Brasil envolve uma privatização ampla, incluindo os serviços infra-estruturais e a telefonia básica. Coutinho, Cassiolato \& Silva (1995, p. 32-36) contrastam esse modelo com outros (praticados na OCDE) que mantêm o setor público atuante, inclusive para prestar serviços de alto valor agregado, de forma a preservar o objetivo de universalização acesso aos serviços básicos.

O modelo de privatização do setor de telecomunicações no Brasil, ao optar por dividir a Telebrás, implementa uma linha de fragmentação que contrasta com o processo de concentração em curso no mercado mundial. Dividida em treze empresas, a Telebrás perderia capacidade de participar em condições de igualdade em associações com empresas de países mais avançados. $\mathrm{O}$ destino das treze empresas em que a Telebrás se dividirá poderá ser o de meras filiais de companhias telefônicas européias ou norte-americanas (Carta Capital, 18/02/1998, p. 33). Dantas (1996) considera que apenas "teles" do Sul e do

\footnotetext{
${ }^{12}$ Nesse ponto, Coutinho, Cassiolato \& Silva (1995, p. 30-31) apresentam uma discussão de uma área que significa uma fusão entre software e infra-estrutura de telecomunicações: o desenvolvimento das redes de telecomunicação nos setores automobilístico: "apesar de algumas tentativas das montadoras de importar da matriz o modelo de redes, não foi possível viabilizar as ligações entre tais empresas e seus fornecedores e distribuidores sem um alto grau de desenvolvimento local".

${ }^{13}$ Os provedores de acesso à Internet no Brasil reclamam da "precária infra-estrutura de telecomunicações - que transformam a navegação na Internet em um pesadelo", segundo a Gazeta Mercantil (9/03/1998, p. C-3).
} 
Sudeste do Brasil serão objeto de interesse dos grandes conglomerados internacionais (p. 120) e que após a fragmentação da Telebrás o Brasil perderá a possibilidade de atuação mais internacional (p. 130). O que significa uma perda de capacidade de ação estratégica na construção de uma infra-estrutura informacional adequada. Desperdiça-se assim uma arma estratégica importante: o tamanho do mercado brasileiro e seu potencial de crescimento (Hawkins, 1995, p. 75).

Os resultados do modelo de privatização das telecomunicações podem levar a um reforço de características perversas do "modelo brasileiro": a ausência do provimento de serviços básicos para o conjunto da população e a prestação de sofisticados serviços de alto valor agregado para setores empresariais e parcelas da população de alta renda. ${ }^{14}$

A política para as telecomunicações é um indicador de que o Brasil não está se preparando de forma adequada para enfrentar os desafios e aproveitar as oportunidades da EBC. A predominância das políticas de "inserção passiva" na ordem internacional limita a capacidade de atuação do país.

\section{Conclusão}

Este texto tem um caráter introdutório. Aponta a necessidade de pesquisas posteriores. Limita-se a uma resenha da literatura sobre a EBC e conjectura sobre possíveis implicações para o caso brasileiro.

Apesar desse caráter, algumas conclusões podem ser provisoriamente assinaladas.

Em primeiro lugar, em função da revolução das TICs e da emergência de uma $\mathrm{EBC}$ nos países mais avançados, amplia-se o hiato tecnológico entre os países situados na fronteira tecnológica e o resto.

Em segundo lugar, a mudança de paradigmas tecnológicos coloca em cena "janelas de oportunidade" aos países periféricos.

Em terceiro lugar, o aproveitamento dessas oportunidades exige um esforço interno expressivo dos países retardatários, concentrado no desenvolvimento de capacidade de absorção.

Em quarto lugar, o Brasil tem pela frente a tarefa de combinar o processo de amadurecimento de seu sistema nacional de inovação com a construção da infra-estrutura informacional. As características discutidas do presente paradigma elevam essa infra-estrutura à condição de um pré-requisito indispensável para o sistema de inovação.

Esses quatro pontos devem ser contrastados com as políticas em curso no

\footnotetext{
${ }^{14}$ Consultor brasileiro da IDC declarou à Gazeta mercantil que a explosão dos negócios na "infovia" é uma realidade incontestável, mas que o futuro dos provedores de acesso à Internet no Brasil é nebuloso, pois "a população de maior poder aquiaitivoa já é usada na rede. Então, não será fácil conquistar novos usuários domésticos. Hoje, esse negócio é considerado arriscado" (9/03/1998, p. C-3).
} 
país, com destaque ao modelo de privatização das telecomunicações. O cenário não é o mais favorável ao cumprimento das tarefas aqui elencadas.

Possivelmente, essa conjuntura não favorável pode estar exigindo dois níveis de reflexão mais cuidadosa: 1) papel do esforço regional e local; 2) papel do setor público.

Dado os problemas do cenário nacional, cresce o papel dos esforços realizados a nível regional e local: inúmeras iniciativas localizadas podem ser pensadas para construir elementos do sistema de inovação e da infra-estrutura informacional, ao menos como uma forma de oferecer "efeitos demonstração" e referências.

Para o setor público, além de novas tarefas que a emergência da EBC apresenta, no caso brasileiro pode ser necessário enfatizar o papel de contrapeso a um eventual surto de desigualdade e exclusão, dada a possibilidade da ausência de acesso universal conviver com a incorporação de parcelas do país e da sociedade às redes internacionais.

\section{Referências bibliográficas}

ALBUQUERQUE, E. (1996) Sistema nacional de inovação no Brasil: uma análise introdutória a partir de dados disponíveis sobre a ciência e a tecnologia. Revista de Economia Política, v. 16, n. 3.

ALBUQUERQUE, E. (1997) Notas sobre os determinantes tecnológicos do catching up: uma introdução à discussão sobre o papel dos sistemas nacionais de inovação na periferia. Estudos Econômicos, v. 27, n. 2.

ALBUQUERQUE, E. (1998) Produção científica e sistema nacional de inovação. Ensaios FEE, v. 19, n. 1, 1998, pp.156-180.

AMSDEN, A. H. (1989) Asia's next giant: South Korea and late industrialization. New York/Oxford: Oxford University.

ARROW, K. (1962) Economic welfare and the allocation of resources for invention. In: LAMBERTON, D. (ed). Economics of information and knowledge. Harmondsworth: Penguin Books, 1971.

ARROW, K. (1996) Technical information and industrial structure. Industrial and Corporate Change, v. 5, n. 2, pp. 645-652.

CASSIOLATO, J. E. (1992) The role of user-producer relations in innovation and diffusion of new technologies: lessons from Brazil. DPhil Thesis. Brighton: University of Sussex.

CASSIOLATO, J. E. (1997) Learning to use telematics technologies in service firms: lessons from the Brazilian experience. Rio: IE-UFRJ (mimeo).

CASTELLS, M. (1996) The information age: economy, society and culture. Oxford: Blackwell.

CHANDLER JR., A. (1990) Scale and scope: the dynamics of industrial capitalism. Harvard: Belknap.

COUTINHO, L.; CASSIOLATO, J. E.; SILVA, A. L. G. (coord.) (1995) Telecomunicações, globalização e competitividade. Campinas: Papirus.

COUTINHO, L.; FERRAZ, J. C. (coords.) (1994) Estudo sobre a competitividade da indústria brasileira. Campinas: Papirus/UNICAMP. 
COWAN, R.; FORAY, D. (1997) The economics of codification and the diffusion of knowledge. MERIT Research Memorandum 2/97-005, Maastricht.

DANTAS, M. (1996) A lógica do capital-informação. Rio: Contraponto.

DAVID, P. (1992) Knowledge, property, and the dynamics of technological change. World Bank conference on development economics, Washington.

DOSI, G. (1984) Technical change and industrial transformation: the theory and an application to the semiconductor industry. London: Macmillan.

EUROPEAN COMMISSION. (1996) The information society and development: the role of the Europena Union. Brussels: EC.

EUROPEAN COMMISSION. (1994) Science and Technology Report. Brussels: European Commission.

EUROPEAN COMMISSION. (1997) Building the European information society for us all: final policy report of the high-level expert group. Brussels: EC.

EVANS, P.; FRISCHTAK, C. TIGRE, P. (orgs.) (1992) Informática brasileira em transição: política governamental e tendências internacionais nos anos 90 . Rio de Janeiro: UFRJ-IEI.

FORAY, D.; LUNDVALL, B-A. (1996) The knowledge-based economy: from the economics of knowledge to the learning economy. In: OECD. Employment and growth in the knowledge-based economy. Paris: OECD.

FREEMAN, C. (1988) Japan, a new system of innovation. In: DOSI, G.; FREEMAN, C.; NELSON, R.; et al. (eds). Technical change and economic theory. London: Pinter, 1988a, p. 330-348.

FREEMAN, C. (1994) The economics of technical change. Cambridge Journal of Economics, v. 18, n. 1.

FREEMAN, C. (1995) The "National System of Innovation" in historical perspective. Cambridge Journal of Economics, v. 19, n. 1.

FREEMAN, C.; PEREZ, C. (1988) Structural crisis of adjustment: business cycles and investment behaviour. In: DOSI, G.; FREEMAN, C.; NELSON, R.; SILVERBERG, G.; SOETE, L. (eds). Technical change and economic theory. London: Pinter, 1988, p. 38-66.

FREEMAN, C.; SOETE, L. (1997) The economics of industrial innovation. London: Pinter.

HAWKINS, R. (1995) Infra-estrutura de informação e comunicações: ambições globais, realidades regionais. In: COUTINHO, L.; CASSIOLATO, J. E.; SILVA, A. L. G. (coord.) Telecomunicações, globalização e competitividade. Campinas: Papirus.

LEMOS, MAURÍCIO B. (1988) Espaço e capital: um estudo sobre a dinâmica centro X periferia. Campinas: UNICAMP/Instituto de Economia, 1988 (Tese de Doutorado em Economia).

LUCENA, C. (1993) A situação atual e o potencial da área de computação no Brasil. São Paulo: EAESP-FGV, 1993. (Pesquisa Ciência \& Tecnologia no Brasil: uma nova política para um mundo global, coordenada por Schwartzman).

MACHLUP, F. (1962) The production and distribution of knowledge in the United States. Princeton: Princeton University.

MANSELL, R.; WHEN, U. (eds.) (1998) Knowledge societies: information technology for sustainable development. Oxford: Oxford University.

MCT-SEPIN. (1997) Relatório de atividades 1996. Brasília: MCT.

NATIONAL SCIENCE FOUNDATION (1996). Science and Engineering Indicators. Washington: National Science Foundation. 
NELSON, R. (1990) Capitalism as an engine of economic growth. Research Policy, v. 19, n. 1.

NELSON, R. (ed.) (1993). National innovation systems: a comparative analysis. New York, Oxford: Oxford University.

OECD. (1996a) Information infrastructure policies in OECD countries. Paris: OECD.

OECD. (1996b) The knowledge-based economy. Paris: OECD

OECD. (1997a) Global information infrastructure - Global information society (GII$G I S)$ : policy requirements. Paris: OECD.

OECD. (1997b) Industrial competitiveness in the knowledge-based economy: the new role of governments. Paris: OECD.

OECD. (1997c) Information technology outlook. Paris: OECD.

OECD. (1997d) Le secteur du logiciel: un profil statistique pour certains pays de 1'OCDE. Paris: OECD.

PATEL, P.; PAVITT, K. (1995) Patterns of technological activity: their measurement and interpretation. In: STONEMAN, P. (ed.) Handbook of the Economics of Innovation and Technological Change. Oxford: Blackwell.

PONDÉ, J. L. (1993) Competitividade da indústria de software: nota técnica setorial do complexo eletrônico. Campinas: IE/UNICAMP, IEI/UFRJ, FDC, FUNCEX (Pesquisa Estudo da competitividade da indústria brasileira, coordenada por Coutinho e Ferraz).

SCHUMPETER, J. (1942) Capitalismo, socialismo e democracia. Rio de Janeiro: Zahar Editores, 1984.

SMITH, K. (1997) Economic infrastructures and innovation systems. In: EDQUIST, C. (ed.) (1997). Systems of Innovation: technologies, institutions and organizations. London: Pinter.

THOMPSON, J. B. (1995) The media and modernity: a social theory of the media. Cambridge: Polity.

VARIAN, H. (1996) The economics of Internet (http://www.sims.berkeley.edu/resources/ infoecom).

VILLASCHI, A. (1992) The Brazilian national system of innovation: opportunities and constraints for transforming technological dependency. London: University of London/University College of London (Tese de Doutorado em Economia).

WORLD BANK. (1997) World Development Report 1997. Oxford: Oxford University. 